\title{
Maxwell-Lorentz without self-interactions: Conservation of energy and momentum.
}

\author{
Jonathan Gratus ${ }^{1,2,3}$ \\ ${ }^{1}$ Department of Physics, Lancaster University, Lancaster LA1 4YB, United Kingdom, \\ 2 The Cockcroft Institute, Sci-Tech Daresbury, Daresbury WA4 4AD, United Kingdom, \\ ${ }^{3}$ https://orcid.org/0000-0003-1597-6084
}

\begin{abstract}
Since a classical charged point particle radiates energy and momentum it is argued that there must be a radiation reaction force. Here we present an action for the MaxwellLorentz without self interactions model, where each particle only responds to the fields of the other charged particles. The corresponding stress-energy tensor automatically conserves energy and momentum in Minkowski and other appropriate spacetimes. Hence there is no need for any radiation reaction.
\end{abstract}

\section{Introduction}

Over the last century there has been significant scientific work debating how elementary charged particles respond to their own electromagnetic field, and the corresponding question of the electromagnetic mass [1-7]. The standard conclusion is that a charged particle obeys the Abraham-Lorentz-Dirac force. This has well known pathologies. There exist runaway solutions where particles accelerate forever without a force [8]. The run-away solutions can be avoided by using the critical submanifold [9], but this leads to pre-acceleration, where a particle moves before a force is applied. Alternative approaches include using a delay equation [3], the Eliezer-Ford-O'Connell equation [10], considering the Landau-Lifshitz equation as fundamental (and not an approximation) [11], variable mass [12,13], and replacing Maxwell's equation with Born-Infield [14] or Bopp-Podolski [15-20].

There have been several experiments to test the existence both classical and quantum of radiation reaction. These include using intense lasers interacting with counter propagating high energy beams [21], laser-plasma interactions [22] and firing lasers into crystals [23]. Summaries of the theory and experimental evidence quantum and classical radiation reaction can be found in [24]. Currently the results are consistent with a quantum radiation reaction, less so with classical radiation reaction or no radiation reaction. Note that the quantum radiation reaction is not considered in this article. With the new intense laser fields available soon, for example with ELI, it is projected that radiation reaction will not only be detectable, but may dominant the motion of electrons [24-26].

For practical purposes it has often been simpler to ignore any radiation reaction and assume that each particle responds via the Lorentz force to the external electromagnetic field 
and the fields of all the other charged particles. After all in most cases the sum of the fields of the other particles will dominate any radiation reaction. For example Klimontovich [27, Page 42] used such a model to make statistical predictions about the nature of plasmas.

The model where each particle does not respond to its own field is referred to as MaxwellLorentz without self-interaction (ML-SI) [28-30]. The ML-SI model is usually criticised on the grounds that it does not account for the damping force experienced by an accelerated charge. Underlying this criticism is the observation that an accelerating charge radiates and that the energy of this radiation must come from somewhere. Usually it is assumed to the kinetic energy of the charged particle. Likewise the radiation has a momentum and this requires a back-force on the radiating particle.

In this letter it is demonstrated that the ML-SI model does, in fact, conserve the total energy and momentum. As a result, the criticisms above are no longer justified. Whatever energy is required to accelerate the charged particles must be balanced by the energy in the electromagnetic field which is lost. This model makes specific predictions. It predicts that no classical radiation reaction will be observed even in the above experiments where it would otherwise be detectable.

In this model, the universe consists of a finite number of point charges. Each charge's 4-acceleration is determined by the sum of the electromagnetic fields of all the other particles. The approach is covariant so it applies to any spacetime. There is no "external" electromagnetic field. The model is described by an action, or equivalently a Lagrangian. As well as deriving the dynamic equations for the particles and fields, the Lagrangian can also be used to derive the stress-energy tensor. There are two ways of deriving the stressenergy tensor from a Lagrangian. By varying the Lagrangian with respect to the metric one obtains the "Hilbert" stress-energy tensor. However one can use the Noether fields and the Belinfante-Rosenfeld modification to derive the "Belinfante-Rosenfeld" stress-energy tensor.

Since all the fields in this action are dynamic, i.e. there are no external fields, other than gravity, there are three important consequences [31]: The Hilbert and Belinfante-Rosenfeld definitions of the stress-energy tensor are equal, the stress-energy tensor is symmetric and it is covariantly conserved. Thus all Killing symmetries of the spacetime will give rise to conserved currents. This establishes the claim that for Minkowski spacetime, energy, momentum and angular momentum are conserved.

We note that this article does not address the radiation reaction problem as usually stated. "How does single charged point particle behave in an external electromagnetic field?" As stated, the model does not permit an external electromagnetic field. We note in section 3 that a universe with a single particle would not interact with any electromagnetic field and hence undergo geodesic motion. Since we give the Lagrangian and stress-energy tensor for the ML-SI, they are different from the equivalents for the usual radiation reaction. In section 4 these equivalents, given by the first terms of equations (14) and (15), can only be defined away from the point particles as they diverge too quickly as one approaches the worldlines of the particles. 
The letter is organised as follows: We start by giving the Lagrangian of the ML-SI model, the corresponding dynamic equations and the stress-energy tensors. The derivation of these results is placed in the appendix. We then give some predictions of this model. In the next section we list additional consequences of the model. We then conclude.

\section{The self-interaction free model}

Let $M$ be spacetime with background metric $g_{\mu \nu}, \mu, \nu=0,1,2,3$ with signature $(-,+,+,+)$. Consider a collection of $N$ particles $\left\{P_{A}, A=1, \ldots, N\right\}$, with masses $m_{A}$ and charges $q_{A}$. The particles travel along worldlines $C_{A}^{\mu}(\tau)$ with proper time $\ddagger$ parameterisation $\tau$ with $\tau_{A}^{-}<\tau<\tau_{A}^{+}$so that $\S$ the 4-velocity $\dot{C}_{A}^{\mu}(\tau)=\frac{d}{d \tau} C_{A}^{\mu}$ satisfies $\dot{C}_{A}^{\mu} \dot{C}_{\mu}^{A}=-1$. One needs to consider $N$ electromagnetic fields $F_{\mu \nu}^{A}$ which are generated by $N$ potentials $\mathcal{A}_{\mu}^{A}$ so that $F_{\mu \nu}^{A}=\partial_{\mu} \mathcal{A}_{\nu}^{A}-\partial_{\nu} \mathcal{A}_{\mu}^{A}$.

The action depends on the $N$ worldlines $C_{A}^{\mu}(\tau)$, the $N$ fields $\mathcal{A}_{\mu}^{A}$ and the metric $g_{\mu \nu}$. It is given formally by

$$
\begin{aligned}
S\left[C_{A}^{\mu}, \mathcal{A}_{\mu}^{A}, g_{\mu \nu}\right]=\sum_{A=1}^{N} & \frac{m_{A}}{2} \int_{\tau_{A}^{-}}^{\tau_{A}^{+}} \dot{C}_{A}^{\mu}(\tau) \dot{C}_{\mu}^{A}(\tau) d \tau-\left.\sum_{A=1}^{N} \sum_{B, B \neq A} q_{A} \int_{\tau_{A}^{-}}^{\tau_{A}^{+}} \dot{C}_{A}^{\mu}(\tau) \mathcal{A}_{\mu}^{B}\right|_{C_{A}(\tau)} d \tau \\
& -\frac{1}{4} \sum_{A=1}^{N} \sum_{B, B \neq A} \int_{M} F_{\mu \nu}^{A} F_{B}^{\mu \nu} \omega d^{4} x
\end{aligned}
$$

where $\omega=\sqrt{(-\operatorname{det} g)}$ and $\sum_{B, B \neq A}$ refers to the double sum over all $B$ excluding $A$, i.e. $\sum_{B, B \neq A}=\sum_{B=1}^{A-1}+\sum_{B=A+1}^{N}$. We state that (1) is the formal action, since the integral is performed over an infinite region and in general would not give a finite result. However we never directly calculate the action integral. Instead we use distributional methods. We write $S$ in terms of a distributional Lagrangian $L$

$$
S\left[C_{A}^{\mu}, \mathcal{A}_{\mu}^{A}, g_{\mu \nu}\right]=\int_{M} L \omega d^{4} x
$$

where

$$
\begin{aligned}
L=\sum_{A=1}^{N} & \frac{m_{A}}{2} \omega^{-1} \int_{\tau_{A}^{-}}^{\tau_{A}^{+}} \dot{C}_{A}^{\mu} \dot{C}_{\mu}^{A} \delta\left(x-C_{A}(\tau)\right) d \tau \\
& \quad-\sum_{A=1}^{N} \sum_{B, B \neq A} q_{A} \omega^{-1} \int_{\tau_{A}^{-}}^{\tau_{A}^{+}} \dot{C}_{A}^{\mu} \mathcal{A}_{\mu}^{B} \delta\left(x-C_{A}(\tau)\right) d \tau-\frac{1}{4} \sum_{A=1}^{N} \sum_{B, B \neq A} F_{\mu \nu}^{A} F_{B}^{\mu \nu} .
\end{aligned}
$$

$\ddagger$ In cases where the particles do not accelerate to lightlike infinity, $\tau_{A}^{-}=-\infty$ and $\tau_{A}^{+}=\infty$. However we do not want to exclude the case when a particle accelerates to infinity in a finite proper time.

$\S$ We use the summation convention on the spacetime index $\mu=0,1,2,3$, no summation on the particle index $A$. The particle index $A$ is placed either high or low for convenience. 
This is a Schwartz distribution and so must act on a test function $\phi$ which has compact support $\|$. Thus it is the integral

$$
\int_{M} L \omega \phi d^{4} x
$$

which is finite. This assumes that no two worldlines ever intersect. To see this, observe that away from all the particles, there are only the electromagnetic fields $F_{\mu \nu}^{A}$, which are all finite. Thus if $\phi$ has support which does not intersect any worldline, the integral (4) is finite.

Along a particle $P_{A}$, the line integral is clearly bounded. Choose an adapted coordinate system $\left(x^{0}, \ldots, x^{3}\right)$ such that $C_{A}^{0}(\tau)=\tau$ and $C_{A}^{i}(\tau)=0$ for $i=1,2,3$. Let $r \in \mathbb{R}$ be the spatial distance given by $r=\left(\left(x^{1}\right)^{2}+\left(x^{2}\right)^{2}+\left(x^{3}\right)^{2}\right)^{1 / 2}$. Then approximately $F_{\mu \nu}^{A}(r) \approx q_{A} r^{-2}$. That is, it does not go to infinity faster than $r^{-2}$ as $r \rightarrow 0$. However in polar coordinates $\omega=r^{2} \sin \theta$ so $F_{\mu \nu}^{A}(r) \omega$ is bounded. Also $F_{\mu \nu}^{B}(r)$ for $P_{B} \neq P_{A}$ are all bounded so the integral in (4) is bounded.

This contrasts with the standard Lagrangian for electromagnetism which contains $F_{\mu \nu} F^{\mu \nu}$ for a single field. In this case $F_{\mu \nu} F^{\mu \nu} \approx r^{-4}$ which is not defined as a distribution.

Varying $S$ with respect to $C_{A}^{\mu}$ gives the Lorentz force equation for the particle $A$,

$$
m_{A} \frac{D \dot{C}_{A}^{\mu}}{d \tau}=q_{A} \dot{C}_{\nu}^{A} \sum_{B, B \neq A} F_{B}^{\nu \mu}
$$

where $\frac{D}{d \tau} \dot{C}_{A}^{\mu}=\ddot{C}_{A}^{\mu}+\Gamma_{\nu \rho}^{\mu} \dot{C}_{A}^{\nu} \dot{C}_{A}^{\rho}$.

Varying $S$ with respect to $\mathcal{A}_{\mu}^{A}$ gives

$$
\sum_{B, B \neq A} \nabla_{\nu} F_{B}^{\nu \mu}=\sum_{B, B \neq A} q_{B} \omega^{-1} \int_{\tau_{B}^{-}}^{\tau_{B}^{+}} \dot{C}_{B}^{\mu} \delta\left(x-C_{B}(\tau)\right) d \tau
$$

This is equivalent to the electromagnetic field $F_{\mu \nu}^{B}$ being generated by the particle $P_{B}$.

$$
\nabla_{\nu} F_{B}^{\nu \mu}=q_{B} \omega^{-1} \int_{\tau_{B}^{-}}^{\tau_{B}^{+}} \dot{C}_{B}^{\mu} \delta\left(x-C_{B}(\tau)\right) d \tau
$$

Differentiating the Lagrangian density $L \omega$ with respect to the metric

$$
T^{\mu \nu}=2 \omega^{-1} \frac{\partial(L \omega)}{\partial g_{\mu \nu}}=2 \frac{\partial L}{\partial g_{\mu \nu}}+2 L \omega^{-1} \frac{\partial \omega}{\partial g_{\mu \nu}},
$$

gives the "Hilbert" stress-energy tensor distribution

$T^{\mu \nu}=\omega^{-1} \sum_{A=1}^{N} \int_{\tau_{A}^{-}}^{\tau_{A}^{+}} m_{A} \delta\left(x-C_{A}(\tau)\right) \dot{C}_{A}^{\mu} \dot{C}_{A}^{\nu} d \tau+\sum_{A=1}^{N} \sum_{B, B \neq A}\left(F_{A \rho}^{\mu} F_{B}^{\nu \rho}-\frac{1}{4} g^{\mu \nu} F_{A}^{\rho \sigma} F_{\rho \sigma}^{B}\right)$.

$\|$ We can think of the test functions $\phi$ as enabling one to measure the integrated action over a small region of spacetime. The same is true for the test tensors $\phi_{\mu \nu}$ which can be used to measure the components of the stress-energy. It is also worth noting that when performing variations the variation fields $\delta \mathcal{A}_{A}^{\mu}$ and $\delta C_{\mu}^{A}$ also have compact support are therefore test tensors. 
This has to act of a test tensor density $\phi_{\mu \nu}$. It is the same as the "Belinfante-Rosenfeld" stress-energy tensor

$$
\begin{aligned}
T^{\mu}{ }_{\nu}=L & \delta_{\nu}^{\mu}-2 \sum_{A=1}^{N} F_{\nu \rho}^{A} \frac{\partial L}{\partial F_{\mu \rho}^{A}}-\sum_{A=1}^{N} \mathcal{A}_{\nu}^{A} \frac{\partial L}{\partial \mathcal{A}_{\mu}^{A}}+\sum_{A=1}^{N} \dot{C}_{A}^{\mu} \frac{\partial L}{\partial \dot{C}_{A}^{\nu}} \\
& -\sum_{A=1}^{N} \delta_{\nu}^{\mu} \delta\left(x-C_{A}(\tau)\right) \frac{\partial L}{\partial\left(\delta\left(x-C_{A}(\tau)\right)\right)}
\end{aligned}
$$

where we define

$$
\begin{aligned}
\delta\left(x-C_{A}(\tau)\right) \frac{\partial}{\partial\left(\delta\left(x-C_{A}(\tau)\right)\right)} \int_{\tau_{B}^{-}}^{\tau_{B}^{+}} \mathcal{L}\left(C_{B}, \dot{C}_{B}\right) \delta\left(x-C_{B}(\tau)\right) d \tau \\
=\delta_{A B} \int_{\tau_{A}^{-}}^{\tau_{A}^{+}} \mathcal{L}\left(C_{A}, \dot{C}_{A}\right) \delta\left(x-C_{A}(\tau)\right) d \tau
\end{aligned}
$$

for any mechanical Lagrangian $\mathcal{L}\left(C_{A}, \dot{C}_{A}\right)$. Here we have two cases, $\mathcal{L}\left(C_{A}, \dot{C}_{A}\right)=\dot{C}_{A}^{\mu} \dot{C}_{\mu}^{A}$ and $\mathcal{L}\left(C_{A}, \dot{C}_{A}\right)=\dot{C}_{A}^{\mu} \mathcal{A}_{\mu}^{B}$. The last term of (10) may look contrived. However it arises from diffeomorphism invariance of the action. The details are outlined in the appendix.

The stress-energy tensor is a total stress-energy tensor, therefore it has the symmetry of the indices

$$
T^{\mu \nu}=T^{\nu \mu}
$$

and the divergenceless condition, which is also known as being covariantly conserved

$$
\nabla_{\mu} T^{\mu \nu}=0
$$

The derivations of (5)-(13) are given in the appendix.

\section{Predictions of the ML-SI model}

The principle prediction of this model all particles simply undergo the Lorentz force. There is no radiation reaction in their equations of motion. It is possible, however, that the field $\sum_{B, B \neq A} F_{\mu \nu}^{B}$, generated by all the other particles, feeds back onto the original particle, $P_{A}$, to give the impression of a radiation reaction.

Another interesting prediction is for the case when the universe has a non-trivial topology. In Minkowski, Friedmann-Lemaître-Robertson-Walker or many other spacetimes it is impossible for a charged particle $P_{A}$ to interact with its own electromagnetic radiation after it has been produced. By contrast if the universe is a 3-torus times time, then $P_{A}$ will see multiple copies of itself and will respond many times as its radiation goes round the torus. This model predicts that whereas $P_{A}$ will respond multiple times to the radiation of the other particles $P_{B} \neq P_{A}$, it will never respond to its own radiation. The same observation could, in principle, be made in our universe by looking at how charged particles behave near a black 
hole. In this case part of the radiation $F_{\mu \nu}^{A}$ will have the opportunity to interact again with $P_{A}$. However, in the ML-SI model it will not do so.

This contrasts with the case when a charged particle $P_{A}$ sees itself in a perfect electrical conductor. A perfect electrical conductor is, itself, an approximation of a real metal made of electrons and protons. These will respond to the initial charged particle and in turn construct a field which can be seen by $P_{A}$.

This model also predicts that, in a universe with just a single charged particle, there would be no electromagnetic field produced and the particle will simply undergo geodesic motion. In Minkowski spacetime, the value of the single homogeneous field $\mathcal{A}_{\text {hom, } A}^{\mu}$, where $A=1$, is irrelevant as it does not affect the motion of the particle $P_{A}$.

\section{Other Consequences of the ML-SI model}

A type of regularisation: We may think of ML-SI as a regularisation of the Lagrangian. Let $F_{\mu \nu}^{\text {tot }}=\sum_{A=1}^{N} F_{\mu \nu}^{A}$ then for points away from any particles we may replace (3) and (9) with

$$
L=\frac{1}{4} F_{\mu \nu}^{\mathrm{tot}} F_{\mathrm{tot}}^{\mu \nu}-\frac{1}{4} \sum_{A=1}^{N} F_{\mu \nu}^{A} F_{A}^{\mu \nu}
$$

and

$$
T^{\mu \nu}=\left(\frac{1}{2} F_{\text {tot } \rho}^{\mu} F_{\text {tot }}^{\rho \nu}+\frac{1}{8} g^{\mu \nu} F_{\text {tot }}^{\rho \sigma} F_{\rho \sigma}^{\mathrm{tot}}\right)-\sum_{A=1}^{N}\left(\frac{1}{2} F_{A \rho}^{\mu} F_{A}^{\rho \nu}+\frac{1}{8} g^{\mu \nu} F_{A}^{\rho \sigma} F_{\rho \sigma}^{A}\right) .
$$

However (14) and (15) do not extend to the worldlines as each term on the right hand sides diverge as $\approx r^{-4}$ as one approaches the worldline. Thus they are not individually distributions.

Liénard-Wiechart fields: In Minkowski spacetime we can solve Maxwell's equations (7) using the Liénard-Wiechart potentials.

$$
\mathcal{A}_{A}^{\mu}(x)=\mathcal{A}_{\text {hom }, A}^{\mu}+\frac{q_{A} \dot{C}_{A}^{\mu}\left(\tau_{\mathrm{R}}\right)}{\dot{C}_{A}^{\nu}\left(\tau_{\mathrm{R}}\right)\left(x-C_{\nu}^{A}\left(\tau_{\mathrm{R}}\right)\right)},
$$

where $\tau_{\mathrm{R}}$ is the retarded time and $\mathcal{A}_{\text {hom,A }}^{\mu}$ is a solution to the source free Maxwell equation $\nabla_{\nu} F_{\text {hom }, A}^{\mu \nu}=0$ with $F_{\mu \nu}^{\text {hom, } A}=\partial_{\mu} \mathcal{A}_{\nu}^{\text {hom }, A}-\partial_{\nu} \mathcal{A}_{\mu}^{\text {hom }, A}$.

Violation of weak energy condition: We observe that the ML-SI stress-energy tensor does not satisfy the weak energy condition. In Minkowski spacetimes, let the particle pass $P_{A}$ through the origin, $C_{A}^{\mu}\left(\tau_{0}\right)=(0,0,0,0)$ with $\dot{C}_{A}^{\mu}\left(\tau_{0}\right)=(1,0,0,0)$. Consider an observer at the point $x^{\mu}=\left(r, x^{1}, x^{2}, x^{3}\right)$ where $r=\left(\left(x^{1}\right)^{2}+\left(x^{2}\right)^{2}+\left(x^{3}\right)^{2}\right)^{1 / 2}$ is small. In this case $T_{\mu \nu} \dot{C}_{A}^{\mu} \dot{C}_{A}^{\nu}=T^{00}=\boldsymbol{E}_{A} \cdot \overline{\boldsymbol{E}}_{A}+\boldsymbol{B}_{A} \cdot \overline{\boldsymbol{B}}_{A}$ where $\boldsymbol{E}_{A}$ and $\boldsymbol{B}_{A}$ are the electric and magnetic fields due to particle $P_{A}$ and $\overline{\boldsymbol{E}}_{A}=\sum_{B, B \neq A} \boldsymbol{E}_{B}$ and $\overline{\boldsymbol{B}}_{A}=\sum_{B, B \neq A} \boldsymbol{B}_{B}$ are the fields due to all the other particles. Now $T^{00}$ is dominated by the Coulomb term, $T^{00} \approx-q_{A} r^{-2} \hat{\boldsymbol{r}} \cdot \overline{\boldsymbol{E}}_{A}$. Now choosing $\boldsymbol{r}= \pm \boldsymbol{E}_{A}$, depending on the sign of $q_{A}$, then $T^{00}<0$. The violation of the weak energy condition does not cause problems for the classical theory, but may cause issues 
for the corresponding quantum theory. This violation is also true for other classical theories such as Bopp-Podolski [17].

The Cauchy problem: Unlike the Abraham-Lorentz-Dirac equation, the initial data for each particle, requires only position and velocity. This suggests the prescribing the Cauchy data should be easier. The Cauchy problem associated ML-SI has been studied in detail [30]. However, in contrast to the continuous source, it is still challenging to define Cauchy data for discrete point particles, even when there is no self-interaction.

\section{Discussion and Conclusion}

In this letter we have presented the case why Maxwell-Lorentz without self interaction is the best model for the dynamics of charged particles. It has many advantages.

The action (1) is a total action, that is all the fields are dynamic. As a result all the equations of motion are derivable from the action and there are no requirements for constitutive relations or equations of state. Another advantage is that the stress-energy tensor derived from varying the Lagrangian with respect to the metric is equivalent to that derived from the Noether current with the Belinfante-Rosenfeld procedure. This tensor is symmetric in its indices and divergenceless and thus gives rise to conserved quantities whenever a Killing vector is present.

The ML-SI makes predictions about the behaviour of electrons in intense electromagnetic fields which is currently being tested.

The Lagrangian is also useful in that it only requires a finite quantity of information to specify, namely the masses and charges of the particles. Although, in general this quantity is large. This contrasts with for example, the cold plasma model of charge, which requires an infinite quantity of information.

This model is certainly simpler than the different models which include radiation reaction. Some of these can also be derived from an action, but which may involve doubling the phase space [32].

The main disadvantage is the violation of the weak stress-energy tensor, which although not relevant for the classical theory, poses challenges for quantisation.

Another criticisms of the ML-SI model is the $N$-fold increase in electromagnetic fields [28]. Thus to set up the Cauchy problem requires $N$ initial conditions for the $N$ particles as well as $N$ initial conditions for the $N$ fields. In Minkowski spacetimes, this can be seen by the $N$ homogeneous fields $\mathcal{A}_{\text {hom, } A}^{\mu}$ given in (16). However if we could argue that one can set the homogeneous fields $\mathcal{A}_{\text {hom, } A}^{\mu}=0$, then we can replace Maxwell's equation (6) with the Liénard-Wiechart fields. Thus we are left with only differential difference equations. Thus the ML-SI approach is similar to the Feynmann-Wheeler theory [33,34], in that particles only communicate with other particles. However since, in the Minkowski case, only the retarded potential is used, the system is manifestly casual.

Even the results of future experiments indicate the existence of classical radiation reaction, the ML-SI model is a useful tool. It may be extended for the interaction of point 
dipoles and quadrupoles [35-38]. Here the multipoles only respond to the electromagnetic fields of the other particles. This is particularly important because the question of the selfinteraction of multipoles is very challenging. Although these multipole models are not derived from a Lagrangian, that they can be described by a function distributional stress-energy tensor is very useful.

\section{Acknowledgements}

The author acknowledges support provided by STFC (Cockcroft Institute, ST/G008248/1 and ST/P002056/1), and EPSRC (Lab in a Bubble, EP/N028694/1). He would also like to thank Dr. David Burton, Dr. Spyridon Talaganis and Alex Warwick for their helpful advice.

\section{References}

[1] Fritz Rohrlich. Classical Charged Particles. Addison Wesley, Reading, Mass, 1965.

[2] J. D. Jackson. Classical Electrodynamics (3rd Edition). Wiley, 1999.

[3] C. Bild, D.-A. Deckert, and H. Ruhl. Radiation reaction in classical electrodynamics. Phys. Rev. D, 99:096001, May 2019.

[4] Mathias Frisch. Inconsistency in classical electrodynamics. Philosophy of Science, 71(4):525-549, 2004.

[5] Stephen Lyle. Self-force and inertia: old light on new ideas, volume 796. Springer, 2010.

[6] Michael R. Ferris and Jonathan Gratus. The origin of the schott term in the electromagnetic self force of a classical point charge. Journal of mathematical physics, 52(9):092902, 2011.

[7] B. P. Kosyakov. Self-interaction in classical gauge theories and gravitation. Physics Reports, 812:1-55, 2019.

[8] Eric Poisson. An introduction to the Lorentz-Dirac equation. arXiv preprint gr-qc/9912045, 1999.

[9] H. Spohn. The critical manifold of the Lorentz-Dirac equation. Europhysics Letters (EPL), 50(3):287-292, may 2000.

[10] J. F. García-Camacho, E. Salinas, A. Avalos-Vargas, and G. Ares de Parga. Mathematical differences and physical similarities between Eliezer-Ford-O'connell equation and Landau-Lifshitz equation. Revista mexicana de física, 61(5):363-371, 2015.

[11] Fritz Rohrlich. The correct equation of motion of a classical point charge. Physics Letters A, 283(56):276-278, 2001.

[12] W. B. Bonnor. A new equation of motion for a radiating charged particle. Proceedings of the Royal Society of London. A. Mathematical and Physical Sciences, 337(1611):591-598, 1974.

[13] Luis Herrera. The equation of motion for a radiating charged particle without self-interaction term. Physics Letters A, 145(1):14-18, 1990.

[14] David A. Burton, Tekin Dereli, and Robin W. Tucker. Born-infeld axion-dilaton electrodynamics and electromagnetic confinement. Physics Letters B, 703(4):530-535, 2011.

[15] Alexei E Zayats. Self-interaction in the Bopp-Podolsky electrodynamics: Can the observable mass of a charged particle depend on its acceleration? Annals of Physics, 342:11-20, 2014.

[16] Jonathan Gratus, Volker Perlick, and Robin W Tucker. On the self-force in Bopp-Podolsky electrodynamics. Journal of Physics A: Mathematical and Theoretical, 48(43):435401, 2015.

[17] R. R. Cuzinatto, C. A. M. de Melo, L. G. Medeiros, B. M. Pimentel, and P. J. Pompeia. Bopp-Podolsky black holes and the no-hair theorem. The European Physical Journal C, 78(1):1-9, 2018.

[18] Michael K.-H. Kiessling. Force on a point charge source of the classical electromagnetic field. Phys. Rev. $D, 100: 065012$, Sep 2019.

[19] Markus Lazar and Jakob Leck. Second gradient electrodynamics: a non-singular relativistic field theory. Annals of Physics, 423:168330, 2020. 
[20] Vu Hoang, Maria Radosz, Angel Harb, Aaron DeLeon, and Alan Baza. Radiation reaction in higher-order electrodynamics. Journal of Mathematical Physics, 62(7):072901, 2021.

[21] J. M. Cole, K. T. Behm, E. Gerstmayr, T. G. Blackburn, J. C. Wood, C. D. Baird, Matthew J. Duff, C. Harvey, A. Ilderton, A. S. Joglekar, et al. Experimental evidence of radiation reaction in the collision of a high-intensity laser pulse with a laser-wakefield accelerated electron beam. Physical Review X, 8(1):011020, 2018.

[22] Kristjan Poder, Matteo Tamburini, G. Sarri, Antonino Di Piazza, S. Kuschel, C. D. Baird, K. Behm, S. Bohlen, J. M. Cole, D. J. Corvan, et al. Experimental signatures of the quantum nature of radiation reaction in the field of an ultraintense laser. Physical Review X, 8(3):031004, 2018.

[23] Tobias N Wistisen, Antonino Di Piazza, Helge V Knudsen, and Ulrik I Uggerhøj. Experimental evidence of quantum radiation reaction in aligned crystals. Nature communications, 9(1):1-6, 2018.

[24] David A. Burton and Adam Noble. Aspects of electromagnetic radiation reaction in strong fields. Contemporary Physics, 55(2):110-121, 2014.

[25] Yevgen Kravets, Adam Noble, and Dino Jaroszynski. Radiation reaction effects on the interaction of an electron with an intense laser pulse. Physical Review E, 88(1):011201, 2013.

[26] A. Di Piazza, C. Müller, K. Z. Hatsagortsyan, and Ch H. Keitel. Extremely high-intensity laser interactions with fundamental quantum systems. Reviews of Modern Physics, 84(3):1177, 2012.

[27] Ronald C Davidson and Qin Hong. Physics of intense charged particle beams in high energy accelerators. World Scientific, 2001.

[28] Dustin Lazarovici. Against fields. European Journal for Philosophy of Science, 8(2):145-170, 2018.

[29] Gernot Bauer, D.-A. Dekert, and Detlef Dürr. Maxwell-lorentz dynamics of rigid charges. Communications in Partial Differential Equations, 38(9):1519-1538, 2013.

[30] Dirk-André Deckert and Vera Hartenstein. On the initial value formulation of classical electrodynamics. Journal of Physics A: Mathematical and Theoretical, 49(44):445202, 2016.

[31] Jonathan Gratus, Yuri N. Obukhov, and Robin W. Tucker. Conservation laws and stress-energymomentum tensors for systems with background fields. Annals of Physics, 327(10):2560-2590, 2012.

[32] P.M.V.B. Barone and A.C.R. Mendes. Lagrangian description of the radiation damping. Physics Letters A, 364(6):438-440, 2007.

[33] John Archibald Wheeler and Richard Phillips Feynman. Interaction with the absorber as the mechanism of radiation. Rev. Mod. Phys., 17:157-181, Apr 1945.

[34] Gernot Bauer, D.-A. Deckert, and Detlef Dürr. On the existence of dynamics in wheeler-feynman electromagnetism. Zeitschrift für angewandte Mathematik und Physik, 64(4):1087-1124, 2013.

[35] J. R. Ellis. Electromagnetic fields of moving dipoles and multipoles. Journal of Mathematical Physics, 7(7):1185-1197, 1966.

[36] William G. Dixon. Dynamics of extended bodies in general relativity-ii. moments of the chargecurrent vector. Proceedings of the Royal Society of London. A. Mathematical and Physical Sciences, 319(1539):509-547, 1970.

[37] Jonathan Gratus and Thomas Banaszek. The correct and unusual coordinate transformation rules for electromagnetic quadrupoles. Proceedings of the Royal Society A: Mathematical, Physical and Engineering Sciences, 474(2213):20170652, 2018.

[38] Jonathan Gratus, Paolo Pinto, and Spyridon Talaganis. The distributional stress-energy quadrupole. Classical and Quantum Gravity, 2020.

\section{Appendix A. Proofs of the formulae}

We use the standard results

$$
\frac{\partial \omega}{\partial g_{\mu \nu}}=-\frac{1}{2} \omega^{-1} \frac{\partial(\operatorname{det} g)}{\partial g_{\mu \nu}}=-\frac{1}{2}(\operatorname{det} g) \omega^{-1} g^{\mu \nu}=\frac{1}{2} \omega g^{\mu \nu}
$$


so

$$
\begin{aligned}
\partial_{\mu} \omega & =\partial_{\mu} \sqrt{(-\operatorname{det} g)}=-\frac{1}{2} \omega^{-1} \partial_{\mu}(\operatorname{det} g)=-\frac{1}{2} \omega^{-1}(\operatorname{det} g) g^{\nu \rho} \partial_{\mu} g_{\nu \rho}=\frac{1}{2} \omega g^{\nu \rho} \partial_{\mu} g_{\nu \rho} \\
& =\frac{1}{2} \omega g^{\sigma \nu}\left(\partial_{\mu} g_{\nu \sigma}+\partial_{\nu} g_{\mu \sigma}-\partial_{\sigma} g_{\mu \nu}\right)=\delta_{\rho}^{\nu} \frac{1}{2} \omega g^{\sigma \rho}\left(\partial_{\mu} g_{\nu \sigma}+\partial_{\nu} g_{\mu \sigma}-\partial_{\sigma} g_{\mu \nu}\right)=\omega \delta_{\rho}^{\nu} \Gamma_{\nu \mu}^{\rho} \\
& =\omega \Gamma_{\nu \mu}^{\nu}
\end{aligned}
$$

and

$$
\frac{\partial g^{\rho \sigma}}{\partial g_{\mu \nu}}=-g^{\rho \alpha} \frac{\partial g_{\alpha \beta}}{\partial g_{\mu \nu}} g^{\beta \sigma}=-g^{\rho \alpha}\left(\delta_{\alpha}^{\mu} \delta_{\beta}^{\nu}\right) g^{\beta \sigma}=-g^{\rho \mu} g^{\nu \sigma}
$$

For the following, let $\overline{\mathcal{A}}_{\mu}^{A}=\sum_{B, B \neq A} \mathcal{A}_{\mu}^{B}, \bar{F}_{\mu \nu}^{A}=\partial_{\mu} \overline{\mathcal{A}}_{\mu}^{A}-\partial_{\nu} \overline{\mathcal{A}}_{\mu}^{A}$ then (1) becomes

$$
S=\sum_{A=1}^{N} \int_{\tau_{A}^{-}}^{\tau_{A}^{+}}\left(\frac{m_{A}}{2} \dot{C}_{A}^{\mu} \dot{C}_{\mu}^{A}-\left.q_{A} \dot{C}_{A}^{\mu} \overline{\mathcal{A}}_{\mu}^{A}\right|_{C_{A}(\tau)}\right) d \tau-\frac{1}{4} \sum_{A=1}^{N} \int_{M} F_{\mu \nu}^{A} \bar{F}_{A}^{\mu \nu} \omega d^{4} x .
$$

Proof that varying (1) with respect to $C_{A}$ gives (5). Let $L_{A}=\frac{1}{2} m_{A} \dot{C}_{A}^{\mu} \dot{C}_{\mu}^{A}-\left.q_{A} \dot{C}_{A}^{\mu} \overline{\mathcal{A}}_{\mu}^{A}\right|_{C_{A}(\tau)}$. From the standard Euler-Lagrange formula, from (A.4) we have

$$
\begin{aligned}
0 & =\frac{\partial L_{A}}{\partial C_{A}^{\mu}}-\frac{d}{d \tau}\left(\frac{\partial L_{A}}{\partial \dot{C}_{A}^{\mu}}\right)=\frac{1}{2} m_{A} \dot{C}_{A}^{\nu} \dot{C}_{A}^{\rho} \partial_{\mu} g_{\nu \rho}-q_{A} \dot{C}_{A}^{\nu} \partial_{\mu} \overline{\mathcal{A}}_{\nu}^{A}-\frac{d}{d \tau}\left(m_{A} \dot{C}_{A}^{\nu} g_{\mu \nu}-q_{A} \overline{\mathcal{A}}_{\mu}^{A}\right) \\
& =\frac{1}{2} m_{A} \dot{C}_{A}^{\nu} \dot{C}_{A}^{\rho} \partial_{\mu} g_{\nu \rho}-q_{A} \dot{C}_{A}^{\nu} \partial_{\mu} \overline{\mathcal{A}}_{\nu}^{A}-m_{A} \ddot{C}_{A}^{\nu} g_{\mu \nu}-m_{A} \dot{C}_{A}^{\nu} \dot{C}^{\rho} \partial_{\rho} g_{\mu \nu}+q_{A} \dot{C}^{\rho} \partial_{\rho} \overline{\mathcal{A}}_{\mu}^{A} \\
& =-q_{A} \dot{C}_{A}^{\nu} \bar{F}_{\mu \nu}^{A}-m_{A} \ddot{C}_{A}^{\nu} g_{\mu \nu}+\frac{1}{2} m_{A} \dot{C}_{A}^{\nu} \dot{C}_{A}^{\rho}\left(\partial_{\rho} g_{\mu \nu}+\partial_{\nu} g_{\mu \rho}-\partial_{\mu} g_{\nu \rho}\right) \\
& =-q_{A} \dot{C}_{A}^{\nu} \bar{F}_{\mu \nu}^{A}-m_{A}\left(\ddot{C}_{A}^{\nu} g_{\mu \nu}+\dot{C}_{A}^{\nu} \dot{C}_{A}^{\rho} \Gamma^{\sigma}{ }_{\nu \rho} g_{\mu \sigma}\right) \\
& =q_{A} \dot{C}_{A}^{\nu} \bar{F}_{\nu \mu}^{A}-m_{A}\left(\ddot{C}_{A}^{\nu} g_{\mu \nu}+\dot{C}_{A}^{\sigma} \dot{C}_{A}^{\rho} \Gamma_{\sigma \rho}^{\nu} g_{\mu \nu}\right) \\
& =q_{A} \dot{C}_{A}^{\nu} \bar{F}_{\nu \mu}^{A}-m_{A} g_{\mu \nu} \dot{C}_{A}^{\rho} \nabla_{\rho} \dot{C}_{A}^{\nu}
\end{aligned}
$$

hence (5) follows.

Proof that varying (1) with respect to $\mathcal{A}_{\mu}^{A}$ gives (6). Observe from (A.2)

$$
\begin{aligned}
\partial_{\nu}\left(F_{A}^{\nu \mu} \omega\right) & =\left(\partial_{\nu} F_{A}^{\nu \mu}\right) \omega+F_{A}^{\nu \mu} \partial_{\nu} \omega=\left(\partial_{\nu} F_{A}^{\nu \mu}\right) \omega+F_{A}^{\nu \mu} \Gamma_{\rho \nu}^{\rho} \omega \\
& =\omega\left(\partial_{\nu} F_{A}^{\nu \mu}+F_{A}^{\rho \mu} \Gamma_{\nu \rho}^{\nu}+F_{A}^{\nu \rho} \Gamma_{\nu \rho}^{\mu}\right)=\omega \nabla_{\nu} F_{A}^{\nu \mu} .
\end{aligned}
$$

From (3) we have

$$
\begin{aligned}
L \omega=\sum_{A=1}^{N} & \frac{m_{A}}{2} \int_{\tau_{A}^{-}}^{\tau_{A}^{+}} \dot{C}_{A}^{\mu} \dot{C}_{\mu}^{A} \delta\left(x-C_{A}\right) d \tau-\sum_{A=1}^{N} \sum_{B, B \neq A} q_{B} \int_{\tau_{B}^{-}}^{\tau_{A}^{+}} \dot{C}_{B}^{\mu} \mathcal{A}_{\mu}^{A} \delta\left(x-C_{B}\right) d \tau \\
& -\frac{1}{2} \sum_{A=1}^{N} \sum_{B, B \neq A} \partial_{\mu} \mathcal{A}_{\nu}^{A} F_{B}^{\mu \nu} \omega,
\end{aligned}
$$

hence

$$
0=\frac{\partial(L \omega)}{\partial \mathcal{A}_{\mu}^{A}}-\partial_{\nu} \frac{\partial(L \omega)}{\partial\left(\partial_{\nu} \mathcal{A}_{\mu}^{A}\right)}=-\sum_{B, B \neq A} q_{B} \int_{\tau_{A}^{-}}^{\tau_{A}^{+}} \dot{C}_{B}^{\mu} \delta\left(x-C_{B}\right) d \tau+\partial_{\nu}\left(\sum_{B, B \neq A} F_{B}^{\nu \mu} \omega\right)
$$




$$
\begin{aligned}
& =\sum_{B, B \neq A}\left(-q_{B} \int_{\tau_{A}^{-}}^{\tau_{A}^{+}} \dot{C}_{B}^{\mu} \delta\left(x-C_{B}\right) d \tau+\partial_{\nu}\left(F_{B}^{\nu \mu} \omega\right)\right) \\
& =\sum_{B, B \neq A}\left(-q_{B} \int_{\tau_{A}^{-}}^{\tau_{A}^{+}} \dot{C}_{B}^{\mu} \delta\left(x-C_{B}\right) d \tau+\omega \nabla_{\nu} F_{B}^{\nu \mu}\right) .
\end{aligned}
$$

Proof that (6) implies (7). Sum (6) over all $A$ (and dividing by the number of particles) gives

$$
\begin{aligned}
0 & =\frac{1}{N} \sum_{A=1}^{N} \sum_{B, B \neq A}\left(\nabla_{\nu} F_{B}^{\nu \mu}-\omega^{-1} q_{B} \int_{\tau_{B}^{-}}^{\tau_{B}^{+}} \dot{C}_{A}^{\mu} \delta\left(x-C_{A}(\tau)\right) d \tau\right) \\
& =\sum_{A=1}^{N}\left(\nabla_{\nu} F_{A}^{\nu \mu}-\omega^{-1} q_{A} \int_{\tau_{A}^{-}}^{\tau_{B}^{+}} \dot{C}_{A}^{\mu} \delta\left(x-C_{A}(\tau)\right) d \tau\right) .
\end{aligned}
$$

Subtracting (6) gives (7).

Proof that (8) gives (9). Writing (A.4) with $g_{\mu \nu}$ explicit we have

$$
\begin{aligned}
\omega L=\sum_{A=1}^{N} & \frac{m_{A}}{2} \int_{\tau_{A}^{-}}^{\tau_{A}^{+}} \dot{C}_{A}^{\mu} \dot{C}_{A}^{\nu} g_{\mu \nu} \delta\left(x-C_{A}(\tau)\right) d \tau-\sum_{A=1}^{N} q_{A} \int_{\tau_{A}^{-}}^{\tau_{A}^{+}} \dot{C}_{A}^{\mu} \overline{\mathcal{A}}_{\mu}^{A} \delta\left(x-C_{A}(\tau)\right) d \tau \\
& -\frac{1}{4} \sum_{A=1}^{N} F_{\mu \nu}^{A} \bar{F}_{\rho \sigma}^{A} g^{\mu \rho} g^{\nu \sigma} \omega .
\end{aligned}
$$

When differentiating with respect to $g_{\mu \nu}$ we ignore the constraint $g_{\mu \nu}=g_{\nu \mu}$. I.e. we assume that $g_{\mu \nu}$ is independent of $g_{\nu \mu}$, when $\mu \neq \nu$. Thus

$$
\frac{\partial}{\partial g_{\mu \nu}}\left(\dot{C}_{A}^{\rho} \dot{C}_{A}^{\sigma} g_{\rho \sigma}\right)=\dot{C}_{A}^{\rho} \dot{C}_{A}^{\sigma} \delta_{\rho}^{\mu} \delta_{\sigma}^{\nu}=\dot{C}_{A}^{\mu} \dot{C}_{A}^{\nu}
$$

Hence differentiating the first term of (A.5) gives the first term of (9). The second term of (A.5) in independent of $g_{\mu \nu}$ so does not contribute to $T^{\mu \nu}$.

For the last term

$$
\begin{aligned}
\frac{\partial}{\partial g_{\mu \nu}}\left(F_{\rho \sigma}^{A} \bar{F}_{\alpha \beta}^{A} g^{\rho \alpha} g^{\sigma \beta}\right) & =-F_{\rho \sigma}^{A} \bar{F}_{\alpha \beta}^{A}\left(g^{\rho \mu} g^{\nu \alpha} g^{\sigma \beta}+g^{\rho \alpha} g^{\sigma \mu} g^{\nu \beta}\right) \\
& =-\left(F_{\rho \sigma}^{A} \bar{F}_{A}^{\nu \sigma} g^{\rho \mu}+F_{\rho \sigma}^{A} \bar{F}_{A}^{\rho \nu} g^{\sigma \mu}\right)=-\left(F_{A \sigma}^{\mu} \bar{F}_{A}^{\nu \sigma}+F_{A \rho}^{\mu} \bar{F}_{A}^{\rho \nu}\right) \\
& =-2 F_{A \rho}^{\mu} F^{\nu \rho} .
\end{aligned}
$$

Hence the last term of (A.5) gives

$$
-\frac{1}{2} \frac{\partial}{\partial g_{\mu \nu}}\left(\sum_{A=1}^{N} F_{\rho \sigma}^{A} \bar{F}_{A}^{\rho \sigma} \omega\right)=\omega \sum_{A=1}^{N}\left(F_{A \rho}^{\mu} \bar{F}^{\rho \nu}-\frac{1}{2} F_{\rho \sigma}^{A} \bar{F}_{A}^{\rho \sigma}\right) .
$$

Hence (9). 
Proof that (10) implies (9). Taking each term in (10) in turn. Second term:

$$
-2 \sum_{A=1}^{N} F_{\nu \rho}^{A} \frac{\partial L}{\partial F_{\mu \rho}^{A}}=\sum_{A=1}^{N} F_{\nu \rho}^{A} \bar{F}_{A}^{\mu \rho}
$$

Third term:

$$
\begin{aligned}
-\sum_{A=1}^{N} \mathcal{A}_{\nu}^{A} \frac{\partial L}{\partial \mathcal{A}_{\mu}^{A}} & =\sum_{A=1}^{N} \mathcal{A}_{\nu}^{A} \sum_{B, B \neq A} q_{A} \omega^{-1} \int_{\tau_{A}^{-}}^{\tau_{A}^{+}} \dot{C}_{B}^{\mu} \delta\left(x-C_{A}(\tau)\right) d \tau \\
& =\sum_{A=1}^{N} q_{A} \omega^{-1} \int_{\tau_{A}^{-}}^{\tau_{A}^{+}} \dot{C}_{A}^{\mu} \overline{\mathcal{A}}_{\nu}^{A} \delta\left(x-C_{A}(\tau)\right) d \tau .
\end{aligned}
$$

Fourth term:

$$
\begin{aligned}
\sum_{A=1}^{N} \dot{C}_{A}^{\mu} \frac{\partial L}{\partial \dot{C}_{A}^{\nu}}= & \sum_{A=1}^{N} m_{A} \omega^{-1} \int_{\tau_{A}^{-}}^{\tau_{A}^{+}} \dot{C}_{A}^{\mu} \dot{C}_{\nu}^{A} \delta\left(x-C_{A}(\tau)\right) d \tau \\
& -\sum_{A=1}^{N} q_{A} \omega^{-1} \int_{\tau_{A}^{-}}^{\tau_{A}^{+}} \dot{C}_{A}^{\mu} \overline{\mathcal{A}}_{\nu}^{A} \delta\left(x-C_{A}(\tau)\right) d \tau .
\end{aligned}
$$

Fifth term:

$$
\begin{aligned}
-\sum_{A=1}^{N} \delta_{\nu}^{\mu} \delta\left(x-C_{A}(\tau)\right) \frac{\partial L}{\partial\left(\delta\left(x-C_{A}(\tau)\right)\right)} \\
=-\delta_{\nu}^{\mu} \sum_{A=1}^{N} \frac{m_{A} \omega^{-1}}{2} \int_{\tau_{A}^{-}}^{\tau_{A}^{+}} \dot{C}_{A}^{\mu} \dot{C}_{\mu}^{A} \delta\left(x-C_{A}(\tau)\right) d \tau \\
\quad+\sum_{A=1}^{N} q_{A} \omega^{-1} \int_{\tau_{A}^{-}}^{\tau_{A}^{+}} \dot{C}_{A}^{\mu} \overline{\mathcal{A}}_{\mu}^{A} \delta\left(x-C_{A}(\tau)\right) d \tau
\end{aligned}
$$

Adding these together gives (9).

Proof of (13). Given test functions $\phi_{\nu}$ then for any tensor $\mathcal{T}^{\mu \nu}$ then

$$
\begin{aligned}
\int_{M}\left(\nabla_{\mu} \mathcal{T}^{\mu \nu}\right) \phi_{\nu} \omega d^{4} x \\
\quad=\int_{M}\left(\partial_{\mu} \mathcal{T}^{\mu \nu}\right) \phi_{\nu} \omega d^{4} x+\int_{M} \mathcal{T}^{\rho \nu} \Gamma_{\mu \rho}^{\mu} \phi_{\nu} \omega d^{4} x+\int_{M} \mathcal{T}^{\mu \rho} \Gamma_{\mu \rho}^{\nu} \phi_{\nu} \omega d^{4} x \\
=-\int_{M} \mathcal{T}^{\mu \nu} \partial_{\mu}\left(\phi_{\nu} \omega\right) d^{4} x+\int_{M} \mathcal{T}^{\rho \nu}\left(\partial_{\rho} \omega\right) \phi_{\nu} d^{4} x+\int_{M} \mathcal{T}^{\mu \rho} \Gamma_{\mu \rho}^{\nu} \phi_{\nu} \omega d^{4} x \\
=-\int_{M} \mathcal{T}^{\mu \nu}\left(\partial_{\mu} \phi_{\nu}\right) \omega d^{4} x+\int_{M} \mathcal{T}^{\mu \rho} \Gamma_{\mu \rho}^{\nu} \phi_{\nu} \omega d^{4} x=-\int_{M} \mathcal{T}^{\mu \nu}\left(\nabla_{\mu} \phi_{\nu}\right) \omega d^{4} x
\end{aligned}
$$

For the first term of (9) we have

$$
\int_{M} \nabla_{\mu}\left(\omega^{-1} \sum_{A=1}^{N} \int_{\tau_{A}^{-}}^{\tau_{A}^{+}} m_{A} \delta\left(x-C_{A}(\tau)\right) \dot{C}_{A}^{\mu} \dot{C}_{A}^{\nu} d \tau\right) \phi_{\nu} \omega d^{4} x
$$




$$
\begin{aligned}
& =-\int_{M}\left(\sum_{A=1}^{N} \int_{\tau_{A}^{-}}^{\tau_{A}^{+}} m_{A} \delta\left(x-C_{A}(\tau)\right) \dot{C}_{A}^{\mu} \dot{C}_{A}^{\nu} d \tau\right)\left(\nabla_{\mu} \phi_{\nu}\right) d^{4} x \\
& =-\sum_{A=1}^{N} \int_{\tau_{A}^{-}}^{\tau_{A}^{+}} m_{A} \dot{C}_{A}^{\mu} \dot{C}_{A}^{\nu}\left(\nabla_{\mu} \phi_{\nu}\right) d \tau=-\sum_{A=1}^{N} \int_{\tau_{A}^{-}}^{\tau_{A}^{+}} m_{A} \dot{C}_{A}^{\nu} \frac{D \phi_{\nu}}{d \tau} d \tau \\
& =-\sum_{A=1}^{N} \int_{\tau_{A}^{-}}^{\tau_{A}^{+}} m_{A} \dot{C}_{A}^{\nu}\left(\frac{d \phi_{\nu}}{d \tau}-\Gamma_{\mu \nu}^{\rho} \dot{C}_{A}^{\mu} \phi_{\nu}\right) d \tau \\
& =\sum_{A=1}^{N} \int_{\tau_{A}^{-}}^{\tau_{A}^{+}} m_{A}\left(\ddot{C}_{A}^{\nu}+\Gamma_{\mu \nu}^{\rho} \dot{C}_{A}^{\nu} \dot{C}_{A}^{\mu}\right) \phi_{\nu} d \tau=\sum_{A=1}^{N} \int_{\tau_{A}^{-}}^{\tau_{A}^{+}} m_{A} \frac{D \dot{C}_{A}^{\nu}}{d \tau} \phi_{\nu} d \tau .
\end{aligned}
$$

Hence using the Lorentz force equation (5)

$$
\begin{aligned}
\nabla_{\mu} \sum_{A=1}^{N} \omega^{-1} \int_{\tau_{A}^{-}}^{\tau_{A}^{+}} m_{B} \delta\left(x-C_{A}(\tau)\right) \dot{C}_{A}^{\mu} \dot{C}_{A}^{\nu} d \tau & =\sum_{A=1}^{N} \omega^{-1} \int_{\tau_{A}^{-}}^{\tau_{A}^{+}} m_{A} \frac{D \dot{C}_{A}^{\mu}}{d \tau} \delta\left(x-C_{A}(\tau)\right) d \tau \\
& =\sum_{A=1}^{N} \omega^{-1} \int_{\tau_{A}^{-}}^{\tau_{A}^{+}} q_{A} \dot{C}_{\nu}^{A} \bar{F}_{A}^{\nu \mu} \delta\left(x-C_{A}(\tau)\right) d \tau
\end{aligned}
$$

For the second term of (9) we have

$$
\begin{aligned}
\nabla_{\nu}\left(\bar{F}_{A \rho}^{\mu} F_{A}^{\nu \rho}-\frac{1}{4} g^{\mu \nu} F_{A}^{\rho \sigma} \bar{F}_{\rho \sigma}^{A}\right) \\
\quad=\left(\nabla_{\nu} \bar{F}_{A \rho}^{\mu}\right) F_{A}^{\nu \rho}+\bar{F}_{A \rho}^{\mu}\left(\nabla_{\nu} F_{A}^{\nu \rho}\right)-\frac{1}{4} g^{\mu \nu}\left(\nabla_{\nu} F_{A}^{\rho \sigma}\right) \bar{F}_{\rho \sigma}^{A}-\frac{1}{4} g^{\mu \nu} F_{A}^{\rho \sigma}\left(\nabla_{\nu} \bar{F}_{\rho \sigma}^{A}\right) \\
\quad=\bar{F}_{A \rho}^{\mu}\left(\nabla_{\nu} F_{A}^{\nu \rho}\right)+\left(\nabla_{\nu} \bar{F}_{\sigma \rho}^{A}\right) g^{\mu \sigma} F_{A}^{\nu \rho}-\frac{1}{2} g^{\mu \nu} F_{A}^{\rho \sigma}\left(\nabla_{\nu} \bar{F}_{\rho \sigma}^{A}\right) \\
=\bar{F}_{A \rho}^{\mu}\left(\nabla_{\nu} F_{A}^{\nu \rho}\right)+\frac{1}{2} g^{\mu \sigma}\left(\left(2 \nabla_{\nu} \bar{F}_{\sigma \rho}^{A}\right) F_{A}^{\nu \rho}-F_{A}^{\rho \nu}\left(\nabla_{\sigma} \bar{F}_{\rho \nu}^{A}\right)\right) \\
=\bar{F}_{A \rho}^{\mu}\left(\nabla_{\nu} F_{A}^{\nu \rho}\right)+\frac{1}{2} g^{\mu \sigma}\left(\left(\nabla_{\nu} \bar{F}_{\sigma \rho}^{A}\right) F_{A}^{\nu \rho}+\left(\nabla_{\rho} \bar{F}_{\sigma \nu}^{A}\right) F_{A}^{\rho \nu}+F_{A}^{\nu \rho}\left(\nabla_{\sigma} \bar{F}_{\rho \nu}^{A}\right)\right) \\
=\bar{F}_{A \rho}^{\mu}\left(\nabla_{\nu} F_{A}^{\nu \rho}\right)+\frac{1}{2} g^{\mu \sigma} F_{A}^{\nu \rho}\left(\nabla_{\nu} \bar{F}_{\sigma \rho}^{A}+\nabla_{\rho} \bar{F}_{\nu \sigma}^{A}+\nabla_{\sigma} \bar{F}_{\rho \nu}^{A}\right) \\
=\bar{F}_{A \rho}^{\mu}\left(\nabla_{\nu} F_{A}^{\nu \rho}\right) .
\end{aligned}
$$

Hence from Maxwell (7)

$$
\begin{aligned}
\nabla_{\nu} \sum_{A=1}^{N}\left(\bar{F}_{A \rho}^{\mu} F_{A}^{\nu \rho}-\frac{1}{4} g^{\mu \nu} F_{A}^{\rho \sigma} \bar{F}_{\rho \sigma}^{A}\right) & =\sum_{A=1}^{N} \bar{F}_{A \rho}^{\mu}\left(\nabla_{\nu} F_{A}^{\nu \rho}\right) \\
& =q_{B} \omega^{-1} \int_{\tau_{B}^{-}}^{\tau_{B}^{+}} \dot{C}_{B}^{\rho} \bar{F}_{A \rho}^{\mu} \delta\left(x-C_{B}(\tau)\right) d \tau \\
& =-\sum_{A=1}^{N} q_{B} \omega^{-1} \int_{\tau_{B}^{-}}^{\tau_{B}^{+}} \dot{C}_{\rho}^{B} \bar{F}_{A}^{\rho \mu} \delta\left(x-C_{B}(\tau)\right) d \tau
\end{aligned}
$$

Combining this with (A.6) gives (13). 
Appendix A.1. Diffeomorphism invariance and the Noether formulation of stress-energy tensor

The stress-energy can be derived in two ways from a Lagrangian. Either from variation with respect to the metric (8) or by using the Noether theorem, with the Belinfante-Rosenfeld procedure (10). That these two are the same is due to: one, the action is diffeomorphism invariant and two, there are no background fields, other than the metric. These two conditions imply that the action is a total action, i.e. all the fields it depends on are dynamic. Total actions also lead to the dynamical equations, the index symmetry of the stress-energy tensor (12) and that it is divergenceless (13). As stated in the conclusion, however, such total actions are actually quite rare. The effect of background fields is detailed in [31].

The details of (10) require discussion about diffeomorphisms which is beyond the scope of this article. However (10) has been demonstrated directly above. To see where the last term of (10) originates from it is sufficient to observe that for a partial Lagrangian

$$
S_{A}^{\mathrm{part}}=\int_{\tau_{A}^{-}}^{\tau_{A}^{+}} \mathcal{L}\left(\dot{C}_{A}^{\mu}, C_{A}^{\mu}\right) d \tau
$$

and corresponding distributional Lagrangian

$$
L_{A}^{\mathrm{part}}=\int_{\tau_{A}^{-}}^{\tau_{A}^{+}} \mathcal{L}\left(\dot{C}_{A}^{\mu}, C_{A}^{\mu}\right) \delta\left(x-C_{A}(\tau)\right) d \tau,
$$

then the corresponding stress-energy tensor is given by the fourth term of (10), i.e.

$$
\left(T_{A}^{\mathrm{part}}\right)^{\mu}{ }_{\nu}=\int_{\tau_{A}^{-}}^{\tau_{A}^{+}} \dot{C}_{A}^{\mu} \frac{\partial \mathcal{L}}{\partial \dot{C}_{A}^{\nu}} \delta\left(x-C_{A}(\tau)\right) d \tau
$$

However, the first term of (10) contains the term $L_{A}^{\text {part }} \delta_{\nu}^{\mu}$, which does not contribute to the stress-energy tensor. We observe from (11)

$$
\delta\left(x-C_{A}(\tau)\right) \frac{\partial L_{B}^{\mathrm{part}}}{\partial\left(\delta\left(x-C_{A}(\tau)\right)\right)}=L_{B}^{\mathrm{part}} \delta_{A B} .
$$

Hence the $L_{A}^{\mathrm{part}} \delta_{\nu}^{\mu}$ terms are cancelled by the last term of (10). 